\title{
Subclinical phenotypes of asthma
}

\section{P Bradding DM FRCP*†, RH Green MD FRCP*}

* Institute for Lung Health and Department of Respiratory Medicine, Glenfield Hospital, Leicester, UK

$\dagger$ Department of Infection, Immunity and Inflammation, University of Leicester, Leicester, UK

Address for Correspondence:

Professor P. Bradding

Department of Respiratory Medicine,

Glenfield Hospital,

Leicester,

LE3 9QP, UK

Tel: 01162583998

Fax: 01162502787

e.mail: pbradding @hotmail.com 


\begin{abstract}
Purpose of review : Asthma is a heterogeneous disease. Identification of specific subphenotypes of asthma may further our understanding of pathophysiology and treatment response, leading to the better targeting of both existing and novel anti-asthma therapies. An accurate and comprehensive clinico-pathological classification system therefore remains an important priority for asthma research. The present review discusses the important recent literature in this field.
\end{abstract}

Recent findings: Cluster analysis in patients with severe asthma has suggested the presence of four distinct clinical phenotypes, two with eosinophilic airway inflammation, and two without. Patients with eosinophilic inflammation benefit most from a management strategy targeting the sputum eosinophil count. Molecular phenotying utilizing gene arrays in steroid naïve asthmatic subjects reveals two distinct subgroups (Th2-high and Th2-low) based on the expression of Th2 cytokine genes (IL-5, IL-13) and Th2-responsive genes. The Th2-high group exhibit clinical features typical of patients with eosinophilic disease. Targeting anti-IL-5 therapy to patients with evidence of eosinophilic airway inflammation and recurrent asthma exacerbations markedly reduces the asthma exacerbation rate, but day-to-day asthma symptoms remain unchanged.

Summary: The detailed phenotyping of asthma will allow the successful targeting of existing and novel therapies to those patients most likely to gain benefit.

Key words: Asthma, phenotype, cluster analysis, gene arrays, anti-IL-5 


\section{Introduction}

Asthma has long been recognised as a heterogeneous disease. Identification of specific sub-phenotypes may further our understanding of pathophysiology, treatment response, prognosis and the underlying genetic basis for the disease. An accurate and comprehensive system of classification therefore remains an important priority for asthma research despite the effort of many clinicians over several generations.

A review of the phenotypic characterisation of asthma was published in this journal in 2007 [1]. In brief, we covered the eosinophilic versus neutrophilic pathological phenotypes, discussed refractory asthma as a distinct inflammatory phenotype, with particular focus on the role of TNF $\alpha$ in severe disease, and discussed phenotype-specific asthma management. The purpose of this review is to discuss recent data of relevance to this field and the previous text will not be repeated.

\section{Multi-dimensional phenotyping}

There are numerous classifications of asthma based on proposed aetiology (allergic, nonallergic, occupational), pathology (eosinophilic, non-eosinophilic), severity and physiological parameters (type I brittle, type II brittle) [1]. In an attempt to provide a more integrated classification of asthma taking into account multidimensional parameters, we undertook k-means cluster analysis in three populations of asthmatic patients [2]••. The first group of patients were identified in primary care $(n=184)$, and had predominantly mild to moderate asthma. The second group $(n=187)$ were recruited from the Glenfield Hospital Difficult Asthma Clinic with predominantly refractory asthma, 
while the third group $(n=68)$ had been studied previously with respect to targeting the sputum eosinophil count to reduce asthma exacerbations [3] .

To determine the most appropriate variables for cluster modelling, we first performed principal components analysis of 16 common clinical measurements and identified factors representative of symptoms, atopy/allergy, eosinophilic inflammation, psychological status, and variable airflow obstruction. So that the cluster analysis was not weighted, only one parameter that was representative of each factor was included in the model. These were: atopic status (allergy domain), peak expiratory flow variability measured as amplitude percent mean of the lowest and highest readings over 2 weeks (variable airflow obstruction domain), induced sputum eosinophil count (airway inflammation domain), and a modified Juniper Asthma Control Score to represent symptoms alone (symptoms domain). Psychological status was considered a consequence of the disease and therefore not included as an input parameter. However, performing the cluster analysis with the inclusion of the anxiety score (the parameter with the higher loading coefficient in the principal components analysis) did not alter the structure of the clusters. Gender, age and body mass index did not load significantly on the factor model but were considered significant determinants of the asthma phenotype and were also included. Methacholine $\mathrm{PC}_{20}$, measured using the tidal breathing method was included for the primary care population only, as it had only been measured in $10 \%$ of the refractory patients in cases of diagnostic doubt. 
The results of the study are summarised in figure 1 (need permission). In the primary care population there were three distinct clusters. The first cluster described a subgroup with early-onset atopic asthma, with evidence of airway dysfunction, symptoms, and eosinophilic airway inflammation. Clinically, this cohort was associated with a significantly greater number of previous hospital attendances and asthma exacerbations requiring oral corticosteroids when compared with the other primary-care subgroups. Cluster 2 described an obese subgroup with a female preponderance, evidence of asthma symptoms, and an absence of eosinophilic airway inflammation. The third cluster had very little evidence of active asthma, a low rate of asthma exacerbations, and it is likely that a proportion of these patients did not have asthma at all. This cluster was labelled as "benign asthma".

In the difficult asthma population, 4 distinct clusters were identified (figure 1).

Two of these were similar to those in the primary care population, i.e the early-onset atopic asthma cluster and the obese, non-eosinophilic cluster. The main difference between patients in these clusters in primary versus secondary care was that patients in secondary care had more severe objective markers of disease. In addition, patients in the early-onset atopic group in secondary care had a higher rate of failed clinic appointments compared to the other refractory clusters, suggesting that perhaps with improved concordance, they could be better controlled.

Two further clusters in the severe asthma cohort were specific for severe disease. One of these was a group of patients with refractory eosinophilic disease, with recurrent 
exacerbations, but relatively few symptoms. These patients were described as inflammation predominant although eosinophilic inflammation predominant would be more accurate to remove any confusion with neutrophilic inflammation. The other severe-specific cluster was a group of patients with early onset atopic disease with virtually no eosinophilic inflammation but very high symptom expression.

The importance of identifying these clusters in severe disease with respect to treatment approaches was highlighted by further analysis of a group of refractory asthmatic patients. These patients had been entered previously into a prospective randomised clinical trial comparing standard care versus treatment guided by the sputum eosinophil count on the rate of asthma exacerbations [3]. This study demonstrated that when corticosteroid treatment is guided by the sputum eosinophil count, there is a $60 \%$ reduction in severe asthma exacerbations and a significant reduction in hospital admissions. When cluster analysis was applied to the patients in this study, three distinct clusters matching those described above were identified (eosinophilic inflammation predominant, obese female, early-onset symptom predominant). Cluster-specific analysis revealed that all of the benefit for preventing exacerbations occurred in the eosinophilic inflammation-predominant cohort (3.53 [SD, 1.18] vs. 0.38 [SD, 0.13] exacerbation/patient/yr, $\mathrm{p}=0.002$ ). In addition, sputum-guided therapy allowed successful downtitration of corticosteroid therapy in early-onset symptom-predominant asthma (mean difference, 1,829 $\mu \mathrm{g}$ beclomethasone equivalent/d [95\% confidence interval, 307$349 \mu \mathrm{g}$ ]; $\mathrm{p}=0.02$ ), without compromising asthma control. 
The identification of these clusters is the first major advance in the complex clinicopathological phenotyping of asthma. It will contribute to the tailored treatment of patients with appropriate therapies, and help to avoid the overuse of corticosteroids in patients who do not have corticosteroid responsive disease. The authors believe that this study is a landmark study in the phenotyping of clinical asthma. The challenge now is to identify the factors which lead to the development and sustained expression of these different phenotypes, and to develop novel phenotype-specific therapies.

\section{Molecular Phenotyping}

Gene arrays are a powerful tool for the unbiased examination of gene expression programmes in tissues, and are now being used to examine the gene expression profiles in asthmatic airways. In an initial study, Woodruff and colleagues identified periostin (POSTN), chloride channel regulator 1 (CLCA1), and serpin peptidase inhibitor, clade B, member 2 (SERPINB2) as epithelial genes that were specifically induced in asthma (as compared to both healthy controls and smokers with mild-moderate COPD), and directly regulated by IL-13 in vitro [4]. In a further study, they therefore used this gene expression signature as a surrogate marker of the IL-13 inflammatory pathway [5]••. They hypothesised that this could be used to identify subsets of patients with asthma who differ in terms of the molecular mechanisms underlying their airway pathology, and that these subsets would constitute distinct inflammatory, pathological and clinical phenotypes. 
For the analysis, the researchers used microarray and PCR analyses of airway epithelial brushings from 42 mild-to moderate corticosteroid-naïve asthmatics and 28 healthy controls. Using unsupervised hierarchical clustering, they identified that approximately $50 \%(\mathrm{n}=22)$ of the asthmatic subjects had consistently high expression of Th2 cytokineinduced genes, while the remaining 20 asthmatic subjects were not distinguishable from normal controls [5] •. These observations were validated on bronchial biopsies using quantitiative PCR to detect Th2 cytokine mRNA. IL-5 and IL-13 mRNA expression was markedly increased in the Th2-high cluster compared to the Th2-low cluster or healthy controls. The Th2-low group of asthmatics did not differ from normal controls in their expression of IL-5 or IL-13 mRNA. IL-4 expression was low across all groups which may relate to the instability of IL-4 gene expression as IL-4 mRNA and protein expression is consistently increased in situ in steroid-naïve asthma [6,7]. IL-5 and IL-13 expression were highly correlated. In keeping with the reciprocal balance hypothesis regarding Th1 versus Th2 expression, Th1 cytokine expression was significantly lower in the Th2-high group. These findings were highly reproducible when re-analysed in bronchial biopsy material obtained from a second bronchoscopy a week later. These findings support earlier work looking at IL-13 protein expression bronchial biopsies and induced sputum, with IL-13 detectable in about 50\% of steroid naïve patients [8].

With respect to clinical features, Th2-high and -low asthma were indistinguishable in terms of demographic characteristics, lung function and bronchodilator reversibility. Both groups were atopic, but the Th2 group had more extensive sensitisation. Interestingly, the Th2-high group had significantly worse bronchial hyperesponsiveness, higher serum IgE, 
greater eosinophilia (blood and BAL). The Th2-high group also had evidence of subepithelial fibrosis, a feature of eosinophilic asthma [9], whereas this was not present in Th2-low asthma. Epithelial mucin stores were also significantly increased in Th2-high asthma.

A very important finding was that only the Th2-high group responded clinically to a course of inhaled corticosteroids, with significant improvements in $\mathrm{FEV}_{1}$. These findings are therefore entirely consistent with earlier work showing that patients with noneosinophilic asthma defined by sputum eosinophil count do not respond to inhaled steroids $[9,10]$, and suggests that the Th2-low group identified here, equates to the noneosinophilic phenotype, and the early-onset symptom predominant non-eosinophilic cluster in the study from Haldar and colleagues. This Th2-low, non eosinophilic group therefore defines a group of asthmatics with an unmet clinical need. It also suggests that patients with Th2-low asthma are unlikely to respond to Th2-specific therapy (vide infra).

Another study sought to discriminate asthma phenotypes on the basis of cytokine profiles in bronchoalveolar lavage (BAL) in samples from patients with mild-moderate and severe asthma [11]•. Out of 25 cytokines measured in the BAL samples of 84 patients (41 severe, 43 mild-moderate) using bead-based multiplex immunoassays, 18 were detectable and included in the analysis. Initial unsupervised agglomerative hierarchical clustering delineated four subgroups of asthma that were independent of treatment. One group was said to be enriched for patients with severe asthma, although these only accounted for $60 \%$ of this group compared to $\sim 50 \%$ of the total cohort. This 
cluster had high concentrations of BAL IL-2 and reduced FEV $1, F V$ and bronchodilator response compared to the other clusters. Classification methods for predicting methacholine sensitivity were also developed, and identified 3 distinct hyperresponder classes that varied in $\mathrm{BAL}$ eosinophil count and $\mathrm{PC}_{20}$ methacholine. This study demonstrates that BAL cytokine profiles have potential to identify subgroups of asthma patients, but at this stage raises more questions that it answers.

\section{Phenotype-specific treatment: Anti-IL-5 therapy.}

Because it is clear that not all asthma is the same, it is essential that new treatments for asthma are tested in those patients most likely to respond. In the past this has occurred fortuitously for $\beta_{2}$-agonists, as nearly all clinical trials of asthma have required betaagonist reversibility as an entry criteria, and thus beta-agonist trials are usually favourable. In practice however, $\mathrm{FEV}_{1}$ reversibility of $>12 \%$, a common entry requirement, is only present in $30 \%$ of our difficult asthma patients, and so it is still unclear how much benefit long-acting beta-agonists for example give to patients with poor bronchodilator responses.

For newer immunomodulators, it unlikely that a therapy such as anti-IL-5 which targets eosinophilic inflammation, will be effective in non-eosinophilic disease. The first published trials of anti-IL-5 treatment were disappointing [12-15], but took "all-comers" with asthma, which will have included patients with both eosinophilic and noneosinophilic disease. Anti-IL-5 antibodies were very effective at reducing sputum and blood eosinophil counts, and reduced tissue eosinophils by approximately $60 \%$ [16], but 
asthma symptoms continued unabated, and bronchial hyperresponsiveness did not improve. Furthermore, there was no attenuation of the airway response to experimental allergen challenge, suggesting that eosinophils are also not important for this [13]. It has been argued that a $60 \%$ reduction in eosinophils within the tissue may not be adequate to reduce their pathological effects, but this seems unlikely because the reduction in eosinophils correlates strongly with a reduction in the deposition of tenascin, lumican, and procollagen III in the lamina reticularis [16].

These results with anti-IL-5 suggested that eosinophils do not play an important role in day-to-day asthma symptoms, variable airflow obstruction or hyperresponsiveness. However, it is known that eosinophils represent an excellent biomarker for predicting whether patients will respond to corticosteroids $[9,10]$, predicting which patients are at risk of exacerbations, and for guiding steroid therapy with a view to preventing these events [3]. This suggested that eosinophils might play a role in the pathophysiology of acute exacerbations but the early anti-IL-5 studies were not powered or designed to test this. There was a non-significant trend towards reduced asthma exacerbations in one antiIL-5 trial [15].

In view of these data, we undertook a randomized, placebo-controlled, double-blind parallel group study of anti-IL-5 treatment (Mepolizumab) in patients with severe asthma with evidence of eosinophilic inflammation and at least 2 severe exacerbations in the previous 12 months [17]••. The primary outcome was the number of severe exacerbations over the 12 month treatment phase. 
Patients were eligible if they met the ATS definition of refractory asthma, had a sputum eosinophil count of $>3 \%$ on at least one occasion in the previous 2 years in spite of high dose inhaled or oral corticosteroid therapy, and at least 2 severe asthma exacerbations in the preceding 12 months. Out of 449 patients in our refractory asthma clinic database, $149(33 \%)$ were potentially eligible. Out of 110 patients approached, 63 were assessed for eligibility, and 61 underwent randomisation.

With respect to the primary outcome, there was a marked reduction in the number of exacerbations in the patients receiving anti-IL-5 (2.0 per patient) compared to those receiving placebo (3.4 per patient, $\mathrm{p}=0.02$ ) (figure 2) (need permission). Furthermore, we saw a significant improvement in quality of life score, suggesting that exacerbations have a significant impact of quality of life. Interestingly, there were no differences in lung function, airway hyperresponsiveness or symptoms between the active treatment group and controls. Also of great interest, treatment with oral prednisolone at the end of the study, led to improvements in lung function and symptoms in both the placebo and antiIL-5 groups. This suggests that symptoms and lung function can be disassociated from eosinophilic inflammation, and are improved by corticosteroids through another mechanism. This work therefore indicates that in patients with eosinophilic asthma, eosinophils contribute to the pathophysiology of exacerbations, but not day-to-day asthma. 
A second smaller study of Mepolizumab therapy in patients with persisting sputum eosinophilia in spite of oral steroid therapy was also published at the same time [18]••. Again, this study demonstrated a marked reduction in exacerbations in the anti-IL-5 group, at the same time as extensive steroid tapering. These studies were the subject of rather negative editorial in the same issue of the N Engl J Med [19]. The editorialist suggested that the patients studied represented a minority of asthma patients, and therefore that anti-IL-5 will be of limited use. We disagree strongly. Thirty three percent of our difficult asthma clinic population were eliglible, and the results were remarkable.

Since anti-IL-5 targets exacerbations but not symptoms or lung function, it will be interesting to see if dual therapy with anti-IL-5 and IL-13 targets a broader aspect of clinical features in patients with Th2-driven eosinophilic asthma

\section{Macrolide therapy}

As shown in figure 1, a proportion of patients with severe asthma have non-eosinophilic disease, and it is known that a proportion of these have evidence of neutrophilic inflammation [20]. Using standard clinical measurements such as spirometry and airway challenges, it is not possible to separate eosinophilic from non-eosinophilic asthma. Patients with non-eosinophilic disease do not respond clinically to inhaled corticosteroids, and do not appear to respond well to oral corticosteroids [2,9].

Macrolide antibiotics are of interest as they are known to have anti-neutrophilic properties [21]. Simpson and colleagues therefore performed a randomised double-blind 
placebo controlled trial of clarithromycin therapy for 8 weeks in 46 patients with severe asthma [22]••. In the treated group there were significant reductions in the number of sputum neutrophils and IL-8 concentrations, and a significant improvement in quality of life. When the data were analysed in subgroups with eosinophilic versus non-eosinophilic disease, the benefit was confined to those with non-eosinophilic disease. This supports the view that an anti-neutrophil approach may be beneficial in in non-eosinophilic asthma, and the results of a larger randomised controlled trial of macrolide therapy in severe non-eosinophilic asthma are awaited.

\section{Conclusion}

These studies carry a very important message for the investigation of new therapies. It is clearly essential to choose an outcome that is likely to be responsive to the intervention, and to chose an intervention for an appropriate patient group. For example, in severe asthma, IL-13 is only present in the airways in about $50 \%$ of subjects [23]• . Treating patients with anti-IL-13 when it is already suppressed might be predicted to fail. There is an obsession with using bronchodilator reversibility of $>12 \%$ as an entry criteria in clinical trials of asthma. In our mepolizumab study, mean bronchodilator reversibility was only $9 \%$. Importantly, post hoc analysis of the Mepolizumab group showed that the patients who received the greatest benefit from treatment, were those with an $\mathrm{FEV}_{1}$ bronchodilator response of $<50 \mathrm{ml}[24] \bullet$. So while reversibility is a potentially useful diagnostic marker of asthma, using it as an essential entry criteria leads to recruitment of

a highly specific subset of asthmatics, which will doom some treatments to failure. So for 
future trials of Mepolizumab, we would urge that bronchodilator reversibility is not an essential entry requirement.

In summary, the clinical and molecular phenotyping approaches discussed in this article are a significant advance in our understanding of the pathophysiology of asthma, revealing several distinct subclinical phenotypes, driven by different pathophysiological mechanisms. Clustering methodology to describe phenotypes is likely to become increasingly popular both clinically and at a molecular level, and we envisage that the two types of research will converge so that phenotypes defined rigorously by both clinical and molecular parameters will be achieved. This should help identify reliable biomarkers as well. The challenge now is to target these subphenotypes with appropriate existing and novel therapies. It is no longer helpful to think of asthma as a single disease entity for which one treatment will treat all patients. 


\section{Reference List}

1. Green RH, Brightling CE, Bradding P. The reclassification of asthma based on subphenotypes. Curr Opin Allergy Clin Immunol 2007, 7:43-50.

2. • Haldar P, Pavord ID, Shaw DE, et al. Cluster analysis and clinical asthma phenotypes. Am J Respir Crit Care Med 2008, 178:218-224.

The first study in asthma to use cluster analysis to identify patients with distinct clinicopathological phenotypes. Those with eosinophilic asthma benefit most from a treatment programme guided by the sputum eosinophil count.

3. Green RH, Brightling CE, McKenna S, et al. Asthma exacerbations and eosinophil counts. A randomised controlled trial. Lancet 2002, 360:1715-1721.

4. Woodruff PG, Boushey HA, Dolganov GM, et al. Genome-wide profiling identifies epithelial cell genes associated with asthma and with treatment response to corticosteroids. Proc Natl Acad Sci U S A 2007, 104:15858-15863.

5. •• Woodruff PG, Modrek B, Choy DF, et al. T-helper type 2-driven inflammation defines major subphenotypes of asthma. Am J Respir Crit Care Med 2009, 180:388395.

This study used gene arrays on airway epithelial brushings and quantitative PCR on bronchial biopsies to define two subsets of patients with steroid-naïve asthma characterised by a Th2-high or Th2-low gene signature. The Th2-high group had features compatible with an eosinophilic phenotype, and benefited most from treatment with inhaled corticosteroids. 
6. Ying S, Humbert M, Barkans J, et al. Expression of IL-4 and IL-5 mRNA and protein product by CD4+ and CD8+ T cells, eosinophils, and mast cells in bronchial biopsies obtained from atopic and nonatopic (intrinsic) asthmatics. J.Immunol. $1997,158: 3539-3544$.

7. Bradding P, Roberts JA, Britten KM, et al. Interleukin-4, -5 , and -6 and tumor necrosis factor-alpha in normal and asthmatic airways: evidence for the human mast cell as a source of these cytokines. Am J Respir Cell Mol Biol 1994, 10:471-480.

8. Berry MA, Parker D, Neale N, et al. Sputum and bronchial submucosal IL-13 expression in asthma and eosinophilic bronchitis. J Allergy Clin Immunol 2004, 114:1106-1109.

9. Berry MA, Morgan A, Shaw DE, et al. Pathological features and inhaled corticosteroid response of eosinophilic and non-eosinophilic asthma. Thorax 2007.

10. Green RH, Brightling CE, Woltmann G, et al. Analysis of induced sputum in adults with asthma: identification of subgroup with isolated sputum neutrophilia and poor response to inhaled corticosteroids. Thorax 2002, 57:875-879.

11. - Brasier AR, Victor S, Boetticher G, et al. Molecular phenotyping of severe asthma using pattern recognition of bronchoalveolar lavage-derived cytokines. J Allergy Clin Immunol 2008, 121:30-37.

This study demonstrates that BAL cytokine profiles have potential to identify subgroups of asthma patients 
12. O'Byrne PM. Cytokines or Their Antagonists for the Treatment of Asthma. Chest 2006, 130:244-250.

13. Leckie MJ, ten Brinke A, Khan J, et al. Effects of an interleukin-5 blocking monoclonal antibody on eosinophils, airway hyper-responsiveness, and the late asthmatic response. Lancet 2000, 356:2144-2148.

14. Kips JC, O'Connor BJ, Langley SJ, et al. Effect of SCH55700, a humanized antihuman interleukin-5 antibody, in severe persistent asthma: a pilot study. Am J Respir Crit Care Med 2003, 167:1655-1659.

15. Flood-Page P, Swenson C, Faiferman I, et al. A study to evaluate safety and efficacy of Mepolizumab in patients with moderate persistent asthma. Am J Respir Crit Care Med 2007, 176:1062-1071.

16. Flood-Page P, Menzies-Gow A, Phipps S, et al. Anti-IL-5 treatment reduces deposition of ECM proteins in the bronchial subepithelial basement membrane of mild atopic asthmatics. J Clin Invest 2003, 112:1029-1036.

17. • Haldar P, Brightling CE, Hargadon B, et al. Mepolizumab and exacerbations of refractory eosinophilic asthma. N Engl J Med 2009, 360:973-984.

This study demonstrates that anti-IL-5 therapy reduces the rate of severe exacerbations in patients with severe refractory eosinophilic asthma and a history of previous exacerbations, but does not improve day-to-day symptoms, lung function or bronchial hyperresponsiveness. This study highlights the importance of targeting novel asthma therapies to the appropriate subphenotype. 
18. • Nair P, Pizzichini MM, Kjarsgaard M, et al. Mepolizumab for prednisonedependent asthma with sputum eosinophilia. N Engl J Med 2009, 360:985-993.

This study of Mepolizumab therapy in patients with persisting sputum eosinophilia in spite of oral steroid therapy demonstrated a marked reduction in exacerbations in the anti-IL-5 group, in parallel with extensive steroid tapering.

19. Wenzel SE. Eosinophils in asthma--closing the loop or opening the door? N Engl J Med 2009, 360:1026-1028.

20. Gibson PG, Simpson JL, Saltos N. Heterogeneity of airway inflammation in persistent asthma : evidence of neutrophilic inflammation and increased sputum interleukin-8. Chest 2001, 119:1329-1336.

21. Oishi K, Sonoda F, Kobayashi S, et al. Role of interleukin-8 (IL-8) and an inhibitory effect of erythromycin on IL- 8 release in the airways of patients with chronic airway diseases. Infect Immun 1994, 62:4145-4152.

22. • Simpson JL, Powell H, Boyle MJ, et al. Clarithromycin targets neutrophilic airway inflammation in refractory asthma. Am J Respir Crit Care Med 2008, $177: 148-155$.

This study shows that clarithromycin reduces sputum neutrophilia in refractory neutrophilic asthma but not eosinophilic asthma. This offers hope that macrolide therapy may have useful clinical effects in neutrophilic disease, where response to existing therapies such as inhaled corticosteroids is poor. 
23. - Saha SK, Berry MA, Parker D, et al. Increased sputum and bronchial biopsy IL-13 expression in severe asthma. J Allergy Clin Immunol 2008, 121:685-691.

IL-13 over-expression is only present in about $50 \%$ of patients with severe asthma. IL-13 overexpressers would seem the logical group to target in trials of anti-IL-13 therapy.

24. • Pavord I, Haldar P, Bradding P, Wardlaw AJ. Mepolizumab in refractory eosinophilic asthma. Thorax 2009,in press.

Patients who respond best to anti-IL-5 therapy have a poor bronchodilator response to $\beta_{2^{-}}$ agonists. 


\section{FIGURE LEGENDS}

Figure 1. Summary of asthma phenotypes observed using cluster analysis in a primary care population and a hospital-based severe asthma clinic. Reproduced with permission from reference 2 .

Figure 2. Asthma exacerbations in patients with severe refractory eosinophilic asthma are reduced by approximately $40 \%$ with the administration of anti-IL-5 (Mepolizumab) therapy. The mean number of exacerbations per subject over the course of the 50 -week treatment period was 2.0 in the mepolizumab group, as compared with 3.4 in the placebo group (relative risk, $0.57 ; 95 \%$ confidence interval, 0.32 to $0.92 ; \mathrm{P}=0.02$ ). Reproduced with permission from reference 17. 